\title{
A Mixture Theory Approach for a Power-Law Fluid Flow through a Porous Channel
}

\author{
Jesús A. Puente Angulo, Maria Laura Martins-Costa, Heraldo da Costa Mattos
}

Laboratory of Theoretical and Applied Mechanics (LMTA), Graduate Program in Mechanical Engineering (PGMEC), Universidade Federal Fluminense 24210-240, Niterói, RJ

E-mail: jpuente@id.uff.br, laura@mec.uff.br, heraldo@mec.uff.br

\begin{abstract}
The steady-state saturated flow of an incompressible power-law fluid through a porous channel limited by two impermeable flat plates is modeled using a mixture theory, which considers fluid and porous matrix as superimposed continuous constituents of a binary mixture. After some simplifying assumptions, the mechanical model gives rise to a coupled system of ordinary differential equations that is simulated by employing a Runge-Kutta method coupled with a shooting strategy. Despite the strong nonlinearity of the problem, this simple methodology provides stable and accurate results, for both shear-thinning and shear-thickening behaviors.
\end{abstract}

\section{Introduction}

Non-Newtonian fluid flows through porous media present various important applications among which one could quote biological engineering (e.g. blood flow), petroleum engineering (demanding efficient extraction techniques and enhanced oil recovery techniques) and Hydrogeology (e.g. soil contamination by pollutants). These relevant applications justify the attention devoted to study this field.

In this work a mixture theory model is employed, requiring constitutive assumptions for both the partial stress tensor (analogous to Cauchy stress tensor) and a momentum source that accounts for the momentum interaction between both constituents of the mixture. This source term acts as a drag force and may be reduced to the classical Darcy's law considering some particular hypotheses.

The non-linear two-point boundary-value problem in ordinary differential equations is simulated using a Runge-Kutta method coupled with a shooting technique. This latter consists is an iterative algorithm, which attempts to identify appropriate initial conditions for a related initial value problem that provides the solution to the original boundary value problem. With this strategy, the solution of the problem is reduced to finding the root of a real function. This methodology has been previously validated by comparing its results with the exact solution for a fluid flowing through a plane channel and also with the exact solution for a Newtonian fluid flowing through the permeable wall channel limited by two impermeable flat plates (MartinsCosta et al., 2013).

\section{Mechanical Model}

The mechanical model combines mass and momentum balance equations for the fluid constituent with constitutive assumptions for the power-law fluid constituent. Supposing the solid constituent (that represents the porous matrix) rigid and at rest, it suffices to solve the motion equations for the fluid constituent, which are given by (Atkin and Craine, 1976; Rajagopal and Tao, 1995) 


$$
\begin{aligned}
& \frac{\partial \rho_{F}}{\partial t}+\nabla \cdot\left(\rho_{F} \mathbf{v}_{F}\right)=0 \\
& \rho_{F}\left[\frac{\partial \mathbf{v}_{F}}{\partial t}+\left(\nabla \mathbf{v}_{F}\right) \mathbf{v}_{F}\right]=\nabla \cdot \mathbf{T}_{F}+\mathbf{m}_{F}+\rho_{F} \mathbf{g}
\end{aligned}
$$

where $\rho_{F}=\phi \rho$ represents the fluid constituent mass density (for a fluid fraction $\phi$ and an actual fluid density $\rho$ ), $\mathbf{v}_{F}$ its velocity, $\mathbf{T}_{F}$ the partial stress tensor associated with it and $\mathbf{m}_{F}$ is an interaction force per unit volume acting on it, due to its interaction with the solid constituent of the mixture. The role of the interaction force is more evident if the Principle of Virtual Power is considered (Costa Mattos et al, 1995) and, in this case, there is a precise framework that allows defining thermodynamically admissible constitutive equations. Constitutive relations for both $\mathbf{T}_{F}$ and $\mathbf{m}_{F}$ are required to build the mechanical model. It is important to note that for saturated flows the fluid fraction $(\phi)$ and the permeability are coincident.

Considering a power-law fluid, Cauchy tensor may be stated as $\mathbf{T}=-p \mathbf{I}+2 \eta(\mathbf{D} \cdot \mathbf{D})^{n} \mathbf{D}$ (Bird et al., 1987, Tanner, 2000), in which $p$ is the hydrostatic pressure acting on the fluid, $\eta$ and $n$ are the power-law rheological parameters that characterize the fluid behavior and $\mathbf{D}$ is the strain rate tensor acting on the fluid. It is important to note that the usual power-law equation, given by $\boldsymbol{\tau}=2 \kappa(\ddot{\gamma})^{m-1} \mathbf{D}$ (Slattery, 1999), in which $\kappa$ is a consistency index and $m$ a power-law index, could be recovered, making $\eta=2^{(m-1) / 2} \kappa$ and $n=(m-1) / 2$. Considering the abovestated Cauchy tensor, the partial stress tensor and the momentum source, are given by (MartinsCosta et al., 2000; Costa Mattos et al. 1995)

$$
\begin{aligned}
& \mathbf{T}_{F}=-p \varphi \mathbf{I}+2 \phi \beta\left(\mathbf{D}_{F} \cdot \mathbf{D}_{F}\right)^{n} \mathbf{D}_{F}, \quad \text { with } \beta=\phi \eta \\
& \mathbf{m}_{F}=-\phi \alpha\left\|\mathbf{v}_{F}\right\|^{2 n} \mathbf{v}_{F}, \quad \text { with } \alpha=\hat{\alpha}(\phi, \eta, K, n)=\frac{\phi \eta}{3 K}\left(\frac{4 n+3}{2 n+1}\right)^{2 n+1}\left(\frac{\phi}{6 K}\right)^{n} .
\end{aligned}
$$

Assuming a steady-state flow of an incompressible fluid constituent though a porous channel with height $2 H$, equations (1)-(2) and the no-slip boundary condition give rise to the following boundary-value problem

$$
\begin{aligned}
& \nabla \cdot \mathbf{v}_{F}=0 \\
& \nabla \cdot\left[-p \phi \mathbf{I}+2 \phi \beta\left(\mathbf{D}_{F} \cdot \mathbf{D}_{F}\right)^{n} \mathbf{D}_{F}\right]-\phi \alpha\left\|\mathbf{v}_{F}\right\|^{2 n} \mathbf{v}_{F}+\rho_{F} \mathbf{g}=0 \\
& \mathbf{v}_{F}=0
\end{aligned}
$$

At this point gravitational effects may be neglected and a fully developed steady-state flow may be considered so that the velocity field in equation (3) may be expressed as $\mathbf{v}_{F}=w \mathbf{i}$, so that considering where $w_{\max }$ as the maximum value of $w$, the following system is reached

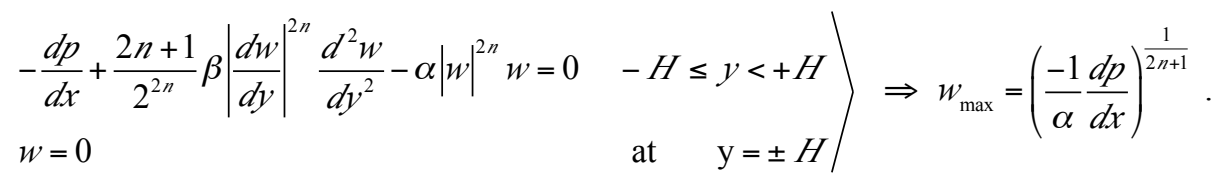




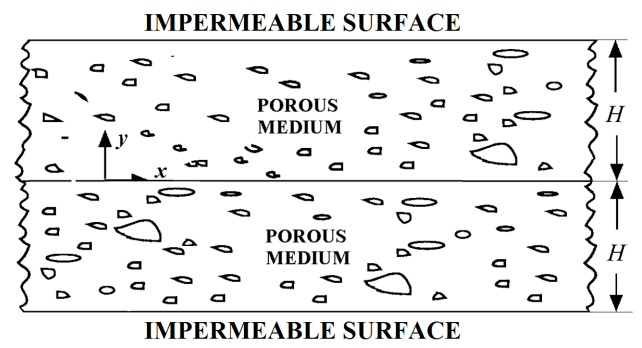

Figure 1: Flow through a plane porous channel.

\section{Numerical Solution}

Equations (4) form a two-point boundary value problem, which can be approximated by using a fourth-order Runge-Kutta methodology coupled with a shooting technique, described below. The theory and practice of the numerical solution of two-point boundary value problems are still very active areas. Although not the first researcher to investigate the solutions of such kind of problem, Keller $(1968 ; 1976)$ was one of the first authors to approach this subject. Those methods were and still are referred to as shooting methods. Several years later, Stoer and Bulirsch (1993) explored shooting techniques in detail. Shooting methods appear to be an interesting way to solve nonlinear boundary value problems. In particular in problems such as

$$
\begin{aligned}
& \frac{d \mathbf{z}}{d y}=\mathbf{f}(y, \mathbf{z}) \quad a \leq y \leq b \\
& \mathbf{h}[\mathbf{z}(a), \mathbf{z}(b)]=\mathbf{0}
\end{aligned}
$$

where $\mathbf{z} \in \mathbb{R}^{n}, \quad \mathbf{f}:[a, b] \rightarrow \mathbb{R}^{n}$ and $h[\mathbf{z}(a) ; \mathbf{z}(b)] \in \mathbb{R}^{m}(m \leq n)$; the related initial value problem can be associated

$$
\begin{aligned}
& \frac{d u}{d y}=\mathbf{f}(y, \mathbf{u}), \quad a \leq y \leq b \\
& \mathbf{u}(a)=s .
\end{aligned}
$$

Then, denoting the solution of Equation (12) by $\mathbf{u} \equiv \mathbf{u}(y ; s)$, we seek $s$ such that

$$
\boldsymbol{\Phi}(s)=\mathbf{h}[s, \mathbf{u}(b ; s)]
$$

If $\hat{s}$ is a root of Equation (7) $(\mathbf{h}[s, \mathbf{u}(b ; s)]=0)$, then $\mathbf{z}(y) \equiv \mathbf{u}(y ; \hat{s})$ is a solution of Equation (5). Conversely, for any solution $\mathbf{z}(u)$ of Equation (5) is a root of Equation (7). Then, the problem is reduced to compute the zero(s) of $\boldsymbol{\Phi}(s)$. It is not difficult to devise existence theorems for Equation (5), but it is not so easy to assure uniqueness. Only the computation of isolated solutions is studied in this paper; the important question of the possibility of multiple solutions is not discussed here.

For the single channel with homogeneous porosity $\phi$, the governing equations described in equation (4) may be conveniently rewritten by considering the change of variables $\mathbf{z}\left(z_{1}, z_{2}\right)$ with $z_{1}=w$ and $z_{2}=d w / d y$, giving rise to the equivalent following system of ordinary differential equations

$$
\frac{d z_{1}}{d y}=z_{2} \quad \text { and } \quad \frac{d z_{2}}{d y}=\frac{2^{2 n}}{(2 n+1) \beta}\left|z_{2}\right|^{-2 n}\left(\frac{d p}{d x}+\alpha\left|z_{1}\right|^{2 n} z_{1}\right)
$$


It is important to note that Equation (8) is valid solely for $z_{2} \neq 0$. However, from Equation (4) it may be verified that when $z_{2}=0 \Rightarrow d w / d y=0$ and the value of $z_{2}$ is defined, since $w|w|^{2 n}=z_{1}\left|z_{1}\right|^{2 n}=-(1 / \alpha)(d p / d x)$. Therefore this relation allows circumventing the limitation of Equation (8) for $z_{2}=0$.

The following boundary-value problem approximates the velocity profile at the porous channel: Find $z_{1}:[-H,+H] \rightarrow \mathbb{R}$ and $z_{2}:[-H,+H] \rightarrow \mathbb{R}$, such that

$$
\begin{gathered}
\frac{d z_{1}}{d y}=z_{2} \\
\frac{d z_{2}}{d y}=\frac{2^{2 n}}{(2 n+1) \beta}\left|z_{2}\right|^{-2 n}\left(\frac{d p}{d x}+\alpha\left|z_{1}\right|^{2 n} z_{1}\right)
\end{gathered} \quad \text { with }\left\{\begin{array}{ccc}
z_{1}=0 & \text { at } & y=-H \\
z_{1}=0 & \text { at } & y=+H
\end{array} .\right.
$$

The problem stated in Equation (9) is equivalent to finding the root of a scalar function represented as $\Phi: \mathbb{R} \rightarrow \mathbb{R} ; s \rightarrow \Phi(s)=z_{1}(y=+H ; s)$, where for a given $s \in \mathbb{R}$, representing an initial estimate, the value $\Phi(s)$ is the value of the variable $z_{1}$ at point $y=+H$, obtained by solving the following initial boundary value problem

$$
\begin{aligned}
& \frac{d z_{2}}{d y}=\frac{2^{2 n}}{(2 n+1) \beta}\left|z_{2}\right|^{-2 n}\left(\frac{d p}{d x}+\alpha\left|z_{1}\right|^{2 n} z_{1}\right) \quad \text { for }-H \leq y<+H ; \\
& \frac{d z_{1}}{d y}=z_{2} \\
& \text { such that }\left\{\begin{array}{rrr}
z_{1}=0 & \text { at } & y=-H \\
z_{2}=t & \text { at } & y=-H
\end{array} .\right.
\end{aligned}
$$

Essentially, this procedure is a shooting technique in which $s$ represents the initial estimate of the derivative $(d w / d y)$ at the point $y=-H$. The initial boundary value problem is approximated by a Runge-Kutta technique (Dahlquist and Bjorc, 1969) and the root of the function $\Phi(s)$ is obtained by an unconditionally convergent procedure, the Bisection method (Dahlquist and Bjorc, 1969). It is important to remark that the above-proposed change of variables is only adequate when $z_{2} \neq 0$.

\section{Numerical Results}

As already expected, for very high permeabilities, present in filter applications, the influence of the Darcian term $(\alpha w)$ - shown in Equation (2) - is almost irrelevant, since as $\alpha \rightarrow 0$ the flow approaches the flow at a channel without a porous matrix, being almost a Stokes flow $\left(d p / d x \approx \beta\left(d^{2} w / d y^{2}\right)\right)$. On the other hand, for small permeabilities, present in important applications as reservoir simulations, the Darcian term is dominant. So, the choice of the numerical methodology to deal with this equation is very important to assure stability and accuracy of the method for all possible parameter values.

It is important to note that $w_{\max }$ occurs exactly when $z_{2}=0$, giving rise to numerical instabilities in a neighborhood of $y=0$, in the process of searching for the root of the function $\Phi(s)$. Due to the behavior of the function $\Phi(s)$, depicted in Figures 2 and 3, Newton-Raphson technique has some convergence shortcomings. Therefore, an unconditionally convergent methodology (such as bisection or regula-falsi) is required to find the root. It is important to 
observe that distinct scales have been employed in the graphs depicted in these figures. The nonlinear nature of $\Phi(s)$ implies that a small variation of the parameter $d w / d y$, for negative values of $n$ causes a very large variation of the parameter $y$, in the vicinity of the root, as shown in Figure 2. A distinct nonlinearity is depicted in Figure 3, when a small variation of the parameter $y$, for $n>0$, in the neighborhood of the root, causes a huge variation of the parameter $d w / d y$. Obviously, the curve $d w / d y$ in the Newtonian case $(n=0)$ is given by a straight line.
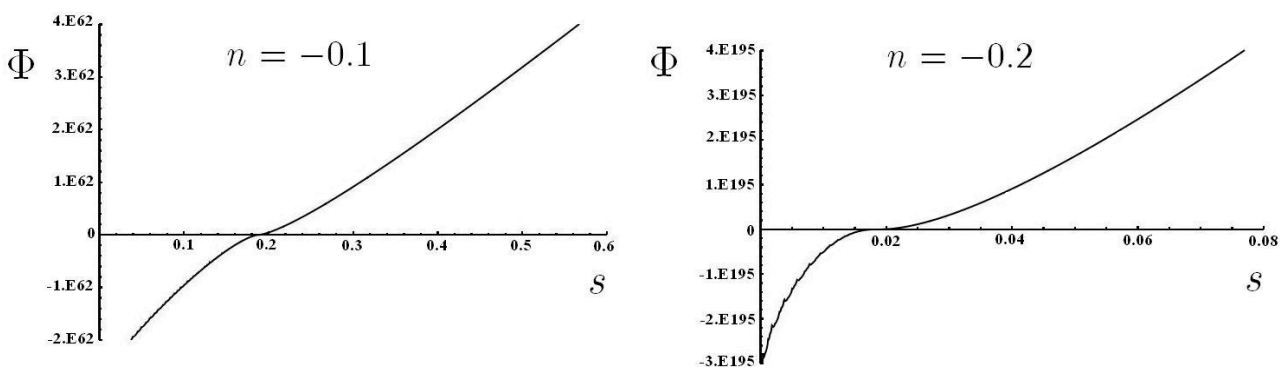

Figure 2: Behavior of function $\Phi(s)$ for negative values of $n$.
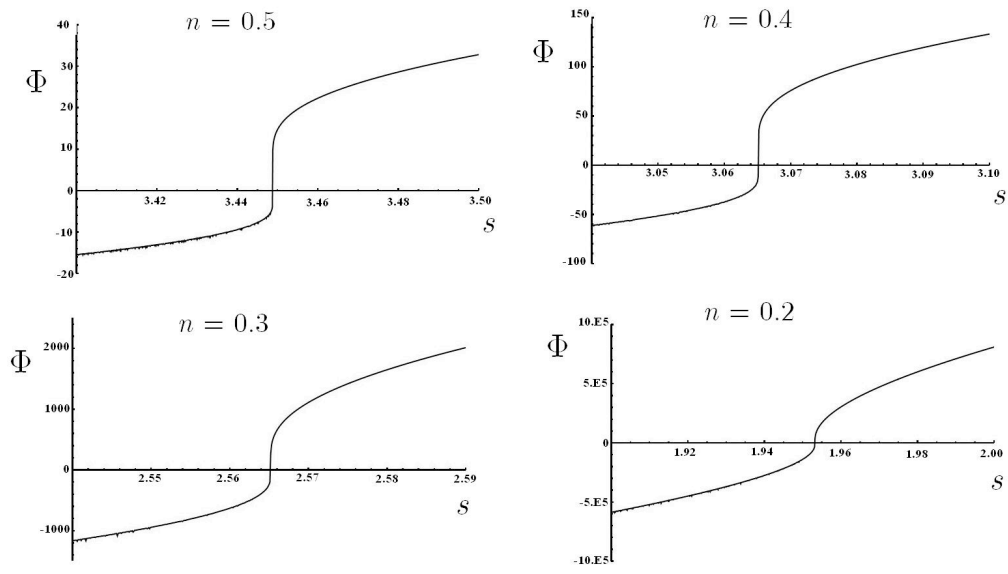

Figure 3: Behavior of function $\Phi(s)$ for positive values of $n$.

As an example, results of flows of a power-law fluid through a porous channel considering shear-thickening and shear-thinning behavior are presented, obtained with the previously described methodology.

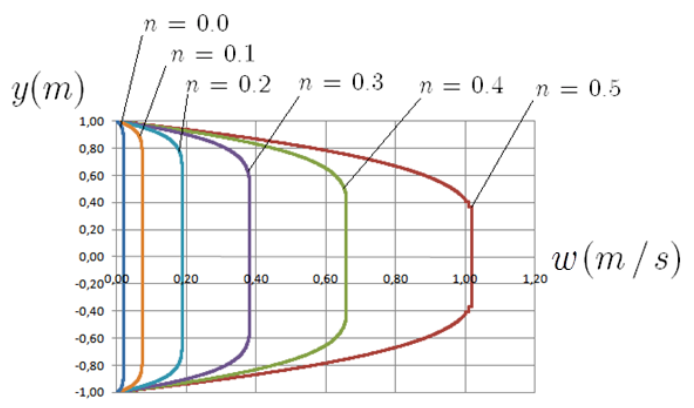

(a)

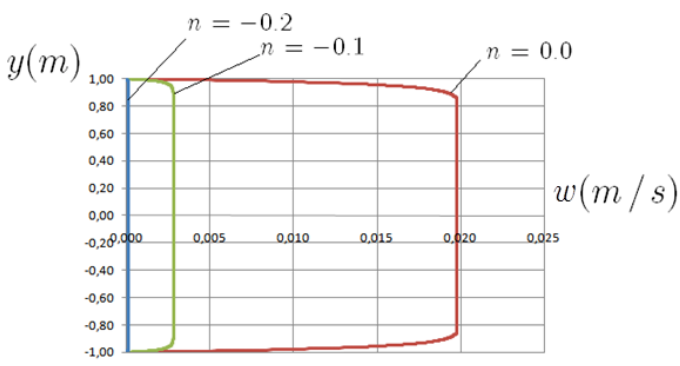

(b)

Figure 4: Flow through a porous channel - velocity profiles: (a) $n \geq 0$; (b) $n \leq 0$. 
Figure 4 shows numerical results for the fluid constituent velocity profile, obtained varying the power-law index $n$ and considering the flow depicted in Figure 1 with the following parameters: $d p / d x=10^{-2} \mathrm{~Pa} / \mathrm{m}, \eta=10^{-3} \mathrm{~Pa} . \mathrm{s}^{\mathrm{n}}, \phi=0.5, \lambda=1, \beta=\phi \eta=0.5 \times 10^{-3} \mathrm{~Pa} . \mathrm{s}^{\mathrm{n}}$ and $K=10^{-3} \mathrm{~m}^{-2}$. It may be noted that the velocity profile becomes flatter as $n$ decreases, in which there is shear-thinning behavior for $n>0$, shear-thickening for $n>0$ and Newtonian for $n=0$. Actually, even employing a distinct scale for negative values of $n$, the velocity profile is almost zero for $n=-0.2$.

Figure 5 shows the behavior of the maximum velocity $w_{\max }$ (in $\mathrm{m} / \mathrm{s}$ ), analytically computed and defined in Equation (4), for distinct values of the power-law index $n$, obtained considering the material parameters stated above and distinct values of the power-law index, showing that it approaches zero for $n<-0.1$. This confirms the behavior depicted in Figure $4 \mathrm{~b}$.

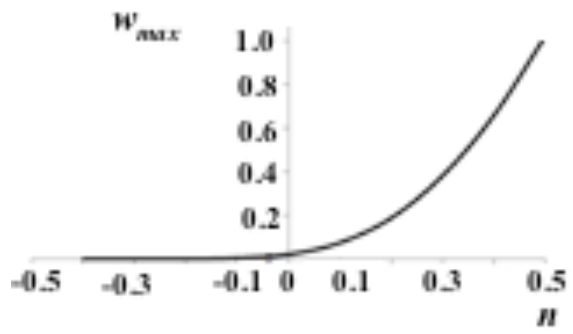

Figure 5: Behavior of the maximum velocity in a porous channel.

It is worth noting that in the methodology presented in this work has been validated by analytical solutions for the flow of a non-Newtonian fluid through a channel without porous matrix and for the flow of a Newtonian fluid through a channel with two distinct adjacent flow regions: one with fluid only and another with this fluid flowing through a porous matrix. (See Martins-Costa et al., 2013). In both cases the numerical solutions and the analytical solutions are indiscernible within the precision of the graphs.

\section{Acknowledgements}

The financial support provided by the Brazilian agency FAPERJ is gratefully acknowledged by the author J. A. Puente Angulo, while the authors M.L. Martins-Costa and H. da Costa Mattos gratefully acknowledge the financial support provided by the Brazilian agency CNPq.

\section{References}

[1] Atkin, R.J. and Craine, R.E., Continuum theories of mixtures. Basic theory and historical development, Quart. J. Mech. Appl. Math., Vol. 29, pp. 209-244 (1976).

[2] Bird, R. B., Armstrong and R. C. Hassager, O., "Dynamics of Polymeric Liquids". v.1, John Wiley \& Sons, U.S.A (1987).

[3] Costa Mattos, H., Martins-Costa, M.L. and Saldanha da Gama, R.M., On the modelling of momentum and energy transfer in incompressible mixtures, Int. J. Non-Linear Mech., Vol. 30, No. 4, pp. 419-431 (1995).

[4] Dahlquist, G. and Bjorck, A., "Numerical Methods", Prentice-Hall, Englewood Cliffs (1969).

[5] Keller, H.B., 1968, "Numerical Methods for Two-Point Boundary-Value Problems", Blaisdell, (1968). 
[6] Keller, H.B., "Numerical Solution of Two Point Boundary Value Problems", SIAM, (1976).

[7] Martins-Costa, M.L., Saldanha da Gama, R.M. and Frey, S., Modelling of a generalized Newtonian flow through channels with permeable wall, Mech. Research Comm., Vol. 27, No. 6, pp. 707-712 (2000).

[8] Martins-Costa, M.L., Puente Angulo, J.A. and Costa Mattos, H.S., Power-law fluid flows in channels with a permeable wall, J. Porous Media, Vol. 16, No. 7, pp. 647-661 (2013).

[9] Rajagopal, K. R. and Tao, L, "Mechanics of Mixtures", Series on Advances in Mathematics for Applied Sciences, Vol. 35, World Scientific, Singapore (1995).

[10] Slattery, J.C., "Advanced Transport Phenomena", Cambridge University Press, USA (1999).

[11] Stoer, J. and Bulirsch, R., "Introduction to Numerical Analysis", Springer-Verlag, second edition, (1993).

[12] Tanner, R. I., "Engineering Rheology”, 2nd ed., Oxford Press, Oxford, UK (2000). 